\title{
Approximating Context-Free Grammars with a Finite-State Calculus
}

\author{
Edmund GRIMLEY EVANS \\ Computer Laboratory \\ University of Cambridge \\ Cambridge, CB2 3QG, GB \\ Edmund.Grimley-Evans@cl.cam.ac.uk
}

\begin{abstract}
Although adequate models of human language for syntactic analysis and semantic interpretation are of at least contextfree complexity, for applications such as speech processing in which speed is important finite-state models are often preferred. These requirements may be reconciled by using the more complex grammar to automatically derive a finite-state approximation which can then be used as a filter to guide speech recognition or to reject many hypotheses at an early stage of processing. A method is presented here for calculating such finite-state approximations from context-free grammars. It is essentially different from the algorithm introduced by Pereira and Wright (1991; 1996), is faster in some cases, and has the advantage of being open-ended and adaptable.
\end{abstract}

\section{Finite-state approximations}

Adequate models of human language for syntactic analysis and semantic interpretation are typically of context-free complexity or beyond. Indeed, Prolog-style definite clause grammars (DCGs) and formalisms such as PATR with feature-structures and unification have the power of Turing machines to recognise arbitrary recursively enumerable sets. Since recognition and analysis using such models may be computationally expensive, for applications such as speech processing in which speed is important finite-state models are often preferred.

When natural language processing and speech recognition are integrated into a single system one may have the situation of a finite-state language model being used to guide speech recognition while a unification-based formalism is used for subsequent processing of the same sentences. Rather than write these two grammars separately, which is likely to lead to problems in maintaining consistency, it would be preferable to derive the finite-state grammar automatically from the (unification-based) analysis grammar.

The finite-state grammar derived in this way can not in general recognise the same language as the more powerful grammar used for analysis, but, since it is being used as a front-end or filter, one would like it not to reject any string that is accepted by the analysis grammar, so we are primarily interested in 'sound approximations' or 'approximations from above'.

Attention is restricted here to approximations of context-free grammars because context-free languages are the smallest class of formal language that can realistically be applied to the analysis of natural language. Techniques such as restriction (Shieber, 1985) can be used to construct context-free approximations of many unification-based formalisms, so techniques for constructing finite-state approximations of context-free grammars can then be applied to these formalisms too.

\section{Finite-state calculus}

A 'finite-state calculus' or 'finite automata toolkit' is a set of programs for manipulating finite-state automata and the regular languages and transducers that they describe. Standard operations include intersection, union, difference, determinisation and minimisation. Recently a number of automata toolkits have been made publicly available, such as FIRE Lite (Watson, 1996), Grail (Raymond and Wood, 1996), and FSA Utilities (van Noord, 1996).

Finite-state calculus has been successfully applied both to morphology (Kaplan and Kay, 1994; Kempe and Karttunen, 1996) and to syntax (constraint grammar, finite-state syntax).

The work described here used a finite-state calculus implemented by the author in SICStus Prolog. 
The use of Prolog rather than $\mathrm{C}$ or $\mathrm{C}++$ causes large overheads in the memory and time required. However, careful account has been taken of the way Prolog operates, its indexing in particular, in order to ensure that the asymptotic complexity is as good as that of the best published algorithms, with the result that for large problems the Prolog implementation outperforms some of the publicly available implementations in $\mathrm{C}++$. Some versions of the calculus allow transitions to be labelled with arbitrary Prolog terms, including variables, a feature that proved to be very convenient for prototyping although it does not essentially alter the power of the machinery. (It is assumed that the string being tested consists of ground terms so no unification is performed, just matching.)

\section{An approximation algorithm}

There are two main ideas behind this algorithm. The first is to describe the finite-state approximation using formulae with regular languages and finite-state operations and to evaluate the formulae directly using the finite-state calculus. The second is to use, in intermediate stages of the calculation, additional, auxiliary symbols which do not appear in the final result. A similar approach has been used for compiling a two-level formalism for morphology (Grimley Evans et al., 1996).

In this case the auxiliary symbols are dotted rules from the given context-free grammar. A dotted rule is a grammar rule with a dot inserted somewhere on the right-hand side, e.g.

$$
\begin{aligned}
& \mathrm{S} \rightarrow \mathrm{NP} \text { VP } \\
& \mathrm{S} \rightarrow \mathrm{NP} \cdot \mathrm{VP} \\
& \mathrm{S} \rightarrow \mathrm{NP} \text { VP. }
\end{aligned}
$$

However, since these dotted rules are to be used as terminal symbols of a regular language, it is convenient to use a more compact notation: they can be replaced by a triple made out of the nonterminal symbol on the left-hand side, an integer to determine one of the productions for that nonterminal, and an integer to denote the position of the dot on the righthand side by counting the number of symbols to the left of the dot. So, if ' $\mathrm{S} \rightarrow \mathrm{NP} V P$ ' is the fourth production for $\mathrm{S}$, the dotted rules given above may be denoted by $\langle S, 4,0\rangle,\langle S, 4,1\rangle$ and $\langle S, 4,2\rangle$, respectively.

It will turn out to be convenient to use a slightly more complicated notation: when the dot is located after the last symbol on the right-hand side we use $z$ as the third element of the triple instead of the corresponding integer, so the last triple is $\langle S, 4, z\rangle$ instead of $\langle S, 4,2\rangle$. (Note that $z$ is an additional symbol, not a variable.) Moreover, for epsilon-rules, where there are no symbols on the right-hand side, we treat the $\epsilon$ as it were a real symbol and consider there to be two corresponding dotted rules, e.g. $\langle M O D, 1,0\rangle$ and $\langle M O D, 1, z\rangle$ corresponding to 'MOD $\rightarrow \cdot \epsilon$ ' and 'MOD $\rightarrow \epsilon$ ' for the rule 'MOD $\rightarrow \epsilon$ '.

Using these dotted rules as auxiliary symbols we can work with regular languages over the alphabet

$$
\begin{aligned}
\Sigma= & T \cup\left\{\langle X, m, n\rangle \mid X \in V \wedge m=1, \ldots, m_{X} \wedge\right. \\
& \left.n=0, \ldots, \max \left\{n_{X, m}-1,0\right\}, z\right\}
\end{aligned}
$$

where $T$ is the set of terminal symbols, $V$ is the set of nonterminals, $m_{X}$ is the number of productions for nonterminal $X$, and $n_{X, m}$ is the number of symbols on the right-hand side of the $m$ th production for $X$.

It will be convenient to use the symbol * as a 'wildcard', so $\langle s, *, 0\rangle$ means $\{\langle X, m, n\rangle \in \Sigma \mid X=$ $s, n=0\}$ and $\langle *, *, z\rangle$ means $\{\langle X, m, n\rangle \in \Sigma \mid n=$ $z\}$. (This last example explains why we use $z$ rather than $n_{X, m}$; it would otherwise not be possible to use the 'wildcard' notation to denote concisely the set $\left\{\langle X, m, n\rangle \mid n=n_{X, m}\right\}$.)

We can now attempt to derive an expression for the set of strings over $\Sigma$ that represent a valid parse tree for the given grammar: the tree is traversed in a top-down left-to-right fashion and the daughters of a node $\mathrm{X}$ expanded with the $m$ th production for $\mathrm{X}$ are separated by the symbols $\langle X, m, *\rangle$. (Equivalently, one can imagine the auxiliary symbols inserted in the appropriate places in the right-hand side of each production so that the grammar is then unambiguous.) Consider, for example, the following grammar:

$$
\begin{aligned}
& S \rightarrow a S b \\
& S \rightarrow \epsilon
\end{aligned}
$$

Then the following is one of the strings over $\Sigma$ that we would like to accept, corresponding to the string $a a b b$ accepted by the grammar:

$$
\begin{gathered}
\langle s, 1,0\rangle a\langle s, 1,1\rangle\langle s, 1,0\rangle a\langle s, 1,1\rangle\langle s, 2,0\rangle\langle s, 2, z\rangle \\
\langle s, 1,2\rangle b\langle s, 1, z\rangle\langle s, 1,2\rangle b\langle s, 1, z\rangle
\end{gathered}
$$

Our first approximation to the set of acceptable strings is $\langle S, *, 0\rangle \Sigma^{*}\langle S, *, z\rangle$, i.e. strings that start with beginning to parse an $S$ and end with having parsed an S. From this initial approximation we subtract (that is, we intersect with the complement of) a series of expressions representing restrictions on the set of acceptable strings: ${ }^{1}$

\footnotetext{
${ }^{1}$ In these expressions over regular languages set union and set difference are denoted by + and -, respectively, while juxtaposition denotes concatenation and the bar denotes complementation $\left(\bar{x} \equiv \Sigma^{*}-x\right)$.
} 


$$
\overline{\left(\Sigma^{*}(\langle *, *, *\rangle-\langle *, *, z\rangle)\right)+\epsilon}\langle *, *, 0\rangle \Sigma^{*}
$$

Formula 1 expresses the restriction that a dotted rule of the form $\langle *, *, 0\rangle$, which represents starting to parse the right-hand side of a rule, may be preceded only by nothing (the start of the string) or by a dotted rule that is not of the form $\langle *, *, z\rangle$ (which would represent the end of parsing the right-hand side of a rule).

$$
\Sigma^{*}\langle *, *, z\rangle \overline{\epsilon+(\langle *, *, *\rangle-\langle *, *, 0\rangle) \Sigma^{*}}
$$

Formula 2 similarly expresses the restriction that a dotted rule of the form $\langle *, *, z\rangle$ may be followed only by nothing or by a dotted rule that is not of the form $\langle *, *, 0\rangle$.

For each non-epsilon-rule with dotted rules $\langle X, m, n\rangle, n=0, \ldots, n_{X, m}-1, z$, for each $n=$ $0, \ldots, n_{X, m}-1$ :

$$
\Sigma^{*}\langle X, m, n\rangle \overline{\operatorname{next}(X, m, n+1) \Sigma^{*}}
$$

where

$$
\begin{array}{cl}
\operatorname{next}(X, m, n)= \\
a\langle X, m, n\rangle & \left(\operatorname{rhs}(X, m, n)=a, a \in T, n<n_{X, m}\right) \\
a\langle X, m, z\rangle & \left(\operatorname{rhs}(X, m, n)=a, a \in T, n=n_{X, m}\right) \\
\langle A, *, 0\rangle & (\operatorname{rhs}(X, m, n)=A, A \in V)
\end{array}
$$

where $\operatorname{rhs}(X, m, n)$ is the $n$th symbol on the righthand side of the $m$ th production for $X$.

Formula 3 states that the dotted rule $\langle X, m, n\rangle$ must be followed by $a\langle X, m, n+1\rangle$ (or $a\langle X, m, z\rangle$ when $\left.n+1=n_{X, m}\right)$ when the next item to be parsed is the terminal $a$, or by $\langle A, *, 0\rangle$ (starting to parse an $A$ ) when the next item is the nonterminal $A$.

For each non-epsilon-rule with dotted rules $\langle X, m, n\rangle, n=0, \ldots, n_{X, m}-1, z$, for each $n=$ $1, \ldots, n_{X, m}-1, z$ :

$$
\overline{\Sigma^{*} \operatorname{prev}(X, m, n)}\langle X, m, n) \Sigma^{*}
$$

where

$$
\begin{array}{lr}
\operatorname{prev}(X, m, n)= & \\
\langle X, m, n-1\rangle a & (\operatorname{rhs}(X, m, n)=a, a \in T, n \neq z) \\
\left\langle X, m, n_{X, m}-1\right\rangle a & (\operatorname{rhs}(X, m, n)=a, a \in T, n=z) \\
\langle A, *, z\rangle \quad(\operatorname{rhs}(X, m, n)=A, A \in V)
\end{array}
$$

Formula 4 similarly states that the dotted rule $\langle X, m, n\rangle$ must be preceded by $\langle X, m, n-1\rangle a$ (or $\left\langle X, m, n_{X, m}-1\right\rangle$ when $\left.n=z\right)$ when the previous item was the terminal $a$, or by $\langle A, *, z\rangle$ when the previous item was the nonterminal $A$.

For each epsilon-rule corresponding to dotted rules $\langle X, m, 0\rangle$ and $\langle X, m, z\rangle$ :

$$
\Sigma^{*}\langle X, m, 0\rangle \overline{\langle X, m, z) \Sigma^{*}}, \text { and }
$$

$$
\overline{\Sigma^{*}\langle X, m, 0\rangle}\langle X, m, z\rangle \Sigma^{*}
$$

Formulae 5 and 6 state that the dotted rule $\langle X, m, 0\rangle$ must be followed by $\langle X, m, z\rangle$, and $\langle X, m, z\rangle$ must be preceded by $\langle X, m, 0\rangle$.

For each non-epsilon rule with dotted rules $\langle X, m, n\rangle, n=0, \ldots, n_{X, m}-1, z$, for each $n=$ $0, \ldots, n_{X, m}-1$ :

$\Sigma^{*}\langle X, m, n\rangle \overline{(\Sigma-\langle X, m, *))^{*}\left(\langle X, m, 0\rangle+\left\langle X, m, n^{\prime}\right\rangle\right) \Sigma^{*}}$

and

$\overline{\Sigma^{*}(\langle X, m, z\rangle+\langle X, m, n\rangle)(\Sigma-\langle X, m, *))^{*}}\left(X, m, n^{\prime}\right\rangle \Sigma^{*}$

where

$$
n^{\prime}= \begin{cases}n+1, & \text { if } n<n_{X, m}-1 \\ z, & \text { if } n=n_{X, m}-1\end{cases}
$$

Formula 7 states that the next instance of $\langle X, m, *\rangle$ that follows $\langle X, m, n\rangle$ must be either $\langle X, m, 0\rangle$ (a recursive application of the same rule) or $\left\langle X, m, n^{\prime}\right\rangle$ (the next stage in parsing the same rule), and there must be such an instance. Formula 8 states similarly that the closest instance of $\langle X, m, *\rangle$ that precedes $\left\langle X, m, n^{\prime}\right\rangle$ must be either $\langle X, m, z\rangle$ (a recursive application of the same rule) or $\langle X, m, n\rangle$ (the previous stage in parsing the same rule), and there must be such an instance.

When each of these sets has been subtracted from the initial approximation we can remove the auxiliary symbols (by applying the regular operator that replaces them with $\epsilon$ ) to give the final finite-state approximation to the context-free grammar.

\section{A small example}

It may be admitted that the notation used for the dotted rules was partly motivated by the possibility of immediately testing the algorithm using the finite-state calculus in Prolog: the regular expressions listed above can be evaluated directly using the 'wildcard' capabilities of the finite-state calculus.

Figure 2 shows the sequence of calculations that corresponds to applying the algorithm to the following grammar:

$$
\begin{aligned}
& \mathrm{S} \rightarrow \mathrm{a} \mathrm{S} \mathrm{b} \\
& \mathrm{S} \rightarrow \epsilon
\end{aligned}
$$

With the following notational explanations it should be possible to understand the code and compare it with the description of the algorithm.

- The procedure $r(R E, X)$ evaluates the regular expression RE and puts the resulting (minimised) automaton into a register with the name $\mathrm{X}$. 
- list_fsa $(X)$ prints out the transition table for the automaton in register $X$.

- Terminal symbols may be any Prolog terms, so the terminal alphabet is implicit. Here atoms are used for the terminal symbols of the gram$\operatorname{mar}(\mathrm{a}$ and $\mathrm{b})$ and terms of the form ${ }_{-} / /_{-}$are used for the triples representing dotted rules. The terms need not be ground, so the Prolog variable symbol _ is used instead of the 'wildcard' symbol * in the description of the algorithm.

- In a regular expression:

- \#X refers to the contents of register $X$;

- \$ represents $\Sigma$, any single terminal symbol;

- $s$ represents a string of terminals with length equal to the number of arguments; so $s$ with no arguments represents the empty string $\epsilon, s(a)$ represents the single terminal $a$, and $s\left(s /{ }_{-} / 0\right)$ represents the dotted rules $\langle s, *, 0\rangle$;

- Kleene star is * (redefined as a postfix operator), and concatenation and union are and + , respectively;

- other operators provided include \& (intersection) and - (difference); there is no operator for complementation; instead subtraction from $\Sigma^{*}$ may be used, e.g. $(\$ *)-(\# I)$ instead of $\bar{L}$;

- rem(RE, L) denotes the result of removing from the language $R E$ all terminals that match one of the expressions in the list L.

The context-free language recognised by the original context-free grammar is $\left\{a^{n} b^{n} \mid n \geq 0\right\}$. The result of applying the approximation algorithm is a 3state automaton recognising the language $\epsilon+a^{+} b^{+}$.

\section{Computational complexity}

Applying the restrictions expressed by formulae 1-6 gives an automaton whose size is at most a small constant multiple of the size of the input grammar. This is because these restrictions apply locally: the state that the automaton is in after reading a dotted rule is a function of that dotted rule.

When restrictions $7-8$ are applied the final automaton may have size exponential in the size of the input grammar. For example, exponential behaviour is exhibited by the following class of grammars:

$$
\begin{aligned}
& \mathrm{S} \rightarrow \mathrm{a}_{1} \mathrm{~S} \mathrm{a}_{1} \\
& \ldots \\
& \mathrm{S} \rightarrow \mathrm{a}_{n} \mathrm{~S} \mathrm{a}_{n} \\
& \mathrm{~S} \rightarrow \epsilon
\end{aligned}
$$

Here the final automaton has $3^{n}$ states. (It records, in effect, one of three possibilities for each terminal symbol: whether it has not yet appeared, has appeared and must appear again, or has appeared and need not appear again.)

There is an important computational improvement that can be made to the algorithm as described above: instead of removing all the auxiliary symbols right at the end they can be removed progressively as soon as they are no longer required; after formulae 7-8 have been applied for each non-epsilon rule with dotted rules $\langle X, m, *\rangle$, those dotted rules may be removed from the finite-state language (which typically makes the automaton smaller); and the dotted rules corresponding to an epsilon production may be removed before formulae 7-8 are applied. (To 'remove' a symbol means to substitute it by $\epsilon$ : a regular operation.)

With this important improvement the algorithm gives exact approximations for the left-linear grammars

$$
\begin{aligned}
& S \rightarrow S a_{1} \\
& \ldots \\
& S \rightarrow S a_{n} \\
& S \rightarrow \epsilon
\end{aligned}
$$

and the right-linear grammars

$$
\begin{aligned}
& S \rightarrow a_{1} S \\
& \cdots \\
& S \rightarrow a_{n} S \\
& S \rightarrow \epsilon
\end{aligned}
$$

in space bounded by $n$ and time bounded by $n^{2}$. (It is easiest to test this empirically with an implementation, though it is also possible to check the calculations by hand.) Pereira and Wright's algorithm gives an intermediate unfolded recogniser of size exponential in $n$ for these right-linear grammars.

There are, however, both left-linear and rightlinear grammars for which the number of states in the final automaton is not bounded by any polynomial function of the size of the grammar. An examples is:

$$
\begin{aligned}
& S \rightarrow a_{1} S \quad S \rightarrow a_{1} A_{1} \\
& \ldots \\
& S \rightarrow a_{n} S \quad S \rightarrow a_{n} A_{n} \\
& \begin{array}{l}
A_{1} \rightarrow a_{1} X \\
A_{2} \rightarrow a_{1} A_{2}
\end{array} \quad \underline{A_{2} \rightarrow a_{2} A_{1} X} \ldots A_{1} \rightarrow a_{n} A_{1} \\
& \ldots \\
& A_{n} \rightarrow a_{1} A_{n} \quad a_{n} A_{2} \\
& X \rightarrow \epsilon
\end{aligned}
$$

Here the grammar has size $\mathrm{O}\left(n^{2}\right)$ and the final approximation has $2^{n+1}-1$ states. 


\begin{tabular}{|c|c|}
\hline MOD $\rightarrow$ & $\mathrm{VP} \rightarrow \mathrm{v} N \mathrm{NP}$ \\
\hline $\mathrm{MOD} \rightarrow \mathrm{p} \mathrm{NP}$ & $\begin{array}{l}\mathrm{VP} \rightarrow \mathrm{v} \mathrm{S} \\
\mathrm{VP} \rightarrow \mathrm{v} \text { VP }\end{array}$ \\
\hline $\mathrm{NOM} \rightarrow$ a NOM & $\mathrm{VP} \rightarrow \mathrm{v}$ \\
\hline $\mathrm{NOM} \rightarrow \mathrm{n}$ & $\mathrm{VP} \rightarrow \mathrm{VP}$ c VP \\
\hline $\mathrm{NOM} \rightarrow$ NOM MOD & $\mathrm{VP} \rightarrow \mathrm{VP} \mathrm{MOD}$ \\
\hline NOM $\rightarrow$ NOM S & $\begin{array}{l}S \rightarrow \text { MOD S } \\
S \rightarrow \text { NP S }\end{array}$ \\
\hline & $\mathrm{S} \rightarrow \mathrm{S}$ c S \\
\hline$\rightarrow \mathrm{d}$ NOM & $\mathrm{S} \rightarrow \mathrm{v}$ NP VP \\
\hline
\end{tabular}

Figure 1: An 18-rule CFG derived from a unification grammar.

Pereira and Wright (1996) point out in the context of their algorithm that a grammar may be decomposed into 'strongly connected' subgrammars, each of which may be approximated separately and the results composed. The same method can be used with the finite-state calculus approach: Define the relation $\mathcal{R}$ over nonterminals of the grammar s.t. $A \mathcal{R} B$ iff $B$ appears on the right-hand side of a production for $A$. Then the relation $\mathcal{S}=\mathcal{R}^{*} \cap\left(\mathcal{R}^{*}\right)^{-1}$, the reflexive transitive closure of $\mathcal{R}$ intersected with its inverse, is an equivalence relation. A subgrammar consists of all the productions for nonterminals in one of the equivalence classes of $\mathcal{S}$. Calculate the approximations for each nonterminal by treating the nonterminals that belong to other equivalence classes as if they were terminals. Finally, combine the results from each subgrammar by starting with the approximation for the start symbol $S$ and substituting the approximations from the other subgrammars in an order consistent with the partial ordering that is induced by $\mathcal{R}$ on the subgrammars.

\section{Results with a larger grammar}

When the algorithm was applied to the 18-rule grammar shown in figure 1 it was not possible to complete the calculations for any ordering of the rules, even with the improvement mentioned in the previous section, as the automata became too large for the finite-state calculus on the computer that was being used. (Note that the grammar forms a single strongly connected component.)

However, it was found possible to simplify the calculation by omitting the application of formulae 7-8 for some of the rules. (The auxiliary symbols not involved in those rules could then be removed before the application of 7-8.) In particular, when restrictions 7-8 were applied only for the S and VP rules the calculations could be completed relatively quickly, as the largest intermediate automaton had only 406 states. Yet the final result was still a useful approximation with 16 states.

Pereira and Wright's algorithm applied to the same problem gave an intermediate automaton (the 'unfolded recogniser') with 56272 states, and the final result (after flattening and minimisation) was a finite-state approximation with 13 states.

The two approximations are shown for comparison in figure 3. Each has the property that the symbols $\mathrm{d}$, $\mathrm{a}$ and $\mathrm{n}$ occur only in the combination $\mathrm{d} \mathrm{a}^{*} \mathrm{n}$. This fact has been used to simplify the state diagrams by treating this combination as a single terminal symbol dan; hence the approximations are drawn with 10 and 9 states, respectively.

Neither of the approximations is better than the other; their intersection (with 31 states) is a better approximation than either. The two approximations have therefore captured different aspects of the context-free language.

In general it appears that the approximations produced by the present algorithm tend to respect the necessity for certain constituents to be present, at whatever point in the string the symbols that 'trigger' them appear, without necessarily insisting on their order, while Pereira and Wright's approximation tends to take greater account of the constituents whose appearance is triggered early on in the string: most of the complexity in Pereira and Wright's approximation of the 18-rule grammar is concerned with what is possible before the first accepting state is encountered.

\section{Comparison with previous work}

Rimon and Herz (1991; 1991) approximate the recognition capacity of a context-free grammar by extracting 'local syntactic constraints' in the form of the Left or Right Short Context of length $n$ of a terminal. When $n=1$ this reduces to next(t), the set of terminals that may follow the terminal $t$. The effect of filtering with Rimon and Herz's next( $t)$ is similar to applying conditions 1-6 from section 3 , but the use of auxiliary symbols causes two differences which can both be illustrated with the following grammar:

$$
\begin{aligned}
& \mathrm{S} \rightarrow \mathrm{a} X \mathrm{X} \mid \mathrm{b} \mathrm{X} \mathrm{b} \\
& \mathrm{X} \rightarrow \epsilon
\end{aligned}
$$

On the one hand, Rimon and Herz's 'next' does not distinguish between different instances of the same terminal symbol, so any $a$, and not just the first one, may be followed by another $a$. On the other hand, Rimon and Herz's 'next' looks beyond the empty constituent in a way that conditions 1-6 do not, so 
$\%$ initial approximation:

$r\left(s\left(s / L_{-} / 0\right)^{-}(\$ *)^{\sim} s\left(s /{ }_{-} / z\right), a\right)$.

$\%$ formulae (1) $-(2)$ :

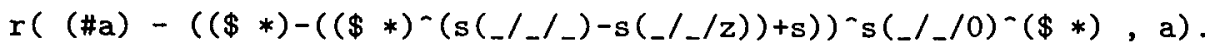

$r\left((\# a)-(\$ *) \sim s\left(I_{-} / z\right) \sim\left((\$ *)-\left(s+\left(s\left(L_{-} / /_{-}\right)-s\left(L_{-} / 0\right)\right)^{-}(\$ *)\right)\right)\right.$, a $)$.

$\%$ formula (3) for "S $\rightarrow$ a $S$ b":

$r\left((\# \mathrm{a})-(\$ *)^{\sim} \mathrm{s}(\mathrm{s} / 1 / 0)^{-}\left((\$ *)-\mathrm{s}(\mathrm{a})^{\sim} \mathrm{s}(\mathrm{s} / 1 / 1)^{-}(\$ *)\right), \mathrm{a}\right)$.

$r\left((\# a)-(\$ *)^{\sim} s(s / 1 / 1)-\left((\$ *)-s\left(s / \_/ 0\right)-(\$ *)\right), a\right)$.

$r\left((\# a)-(\$ *) \sim s(s / 1 / 2) \sim\left((\$ *)-s(b)^{\sim} s(s / 1 / z)^{\sim}(\$ *)\right), a\right)$.

$\%$ formula (4) for "S $\rightarrow$ a $S$ b":

$r\left((\# a)-\left((\$ *)-(\$ *)^{\wedge} s(s / 1 / 0)^{\wedge} s(a)\right)^{\wedge} s(s / 1 / 1)^{\sim}(\$ *), a\right)$.

$r\left((\# a)-\left((\$ *)-(\$ *)^{\wedge} s(s / / / z)\right) \sim s(v p / 2 / 1) \sim(\$ *), a\right)$.

$r\left((\# a)-\left((\$ *)-(\$ *)^{\wedge} s(s / 1 / 2)^{\wedge} s(b)\right)^{\wedge} s(s / 1 / z)^{-}(\$ *), a\right)$.

$\%$ formulae (5)-(6) for "S $\rightarrow$ ":

$r\left((\# a)-(\$ *)^{\wedge} s(s / 2 / 0)^{\sim}\left((\$ *)-s(s / 2 / z)^{-}(\$ *)\right), a\right)$.

$r\left((\# a)-((\$ *)-(\$ *) \sim s(s / 2 / 0))^{\sim} s(s / 2 / z)-(\$ *), a\right)$.

$\%$ formula $(7)$ for "S $\rightarrow$ a S b":

$r\left((\# a)-(\$ *)^{-} s(s / 1 / 0)^{-}((\$ *)-((\$-s(s / 1 / 2)) *)-(s(s / 1 / 0)+s(s / 1 / 1))-(\$ *)), a\right)$.

$\left.r\left((\# a)-(\$ *)^{n} s(s / 1 / 1)^{-}\left((\$ *)-\left(\left(\$-s(s / 1 /)_{-}\right)\right) *\right)^{-}(s(s / 1 / 0)+s(s / 1 / 2))^{-}(\$ *)\right), a\right)$.

$r\left((\# a)-(\$ *)^{\wedge} s(s / 1 / 2)^{\wedge}\left((\$ *)-\left(\left(\$-s\left(s / 1 / \_\right)\right) *\right)^{-}(s(s / 1 / 0)+s(s / 1 / z))^{-}(\$ *)\right), a\right)$.

$\%$ formula (8) for "S $\rightarrow$ a $\mathrm{S} \mathrm{b}$ ":

$r\left((\# a)-\left((\$ *)-(\$ *)-(s(s / 1 / z)+s(s / 1 / 0))-\left(\left(\$-s\left(s / 1 / \_\right)\right) *\right)\right)-s(s / 1 / 1)-(\$ *), a\right)$.

$r\left((\# a)-\left((\$ *)-(\$ *)-(s(s / 1 / z)+s(s / 1 / 1))-\left(\left(\$-s\left(s / 1 / \_\right)\right) *\right)\right)^{-} s(s / 1 / 2)^{-}(\$ *), a\right)$.

$r\left((\# a)-\left((\$ *)-(\$ *)-(s(s / 1 / z)+s(s / 1 / 2))-\left(\left(\$-s\left(s / 1 / \_\right)\right) *\right)\right)^{-} s(s / 1 / z)^{-}(\$ *), a\right)$.

$\%$ define the terminal alphabet:

$r(s(s / 1 / 0)+s(s / 1 / 1)+s(s / 1 / 2)+s(s / 1 / z)+s(s / 2 / 0)+s(s / 2 / z)+s(a)+s(b), s i g m a)$.

$\%$ remove the auxiliary symbols to give final result:

$r\left(\operatorname{rem}\left((\# a) \&((\# \operatorname{sigma}) *),\left[/_{-} /{ }_{-}\right]\right), f\right)$.

list_fsa(f).

Figure 2: The sequence of calculations for approximating $S \rightarrow a \mathrm{~S} \mathrm{~b} \mid \epsilon$, coded for the finite-state calculus.
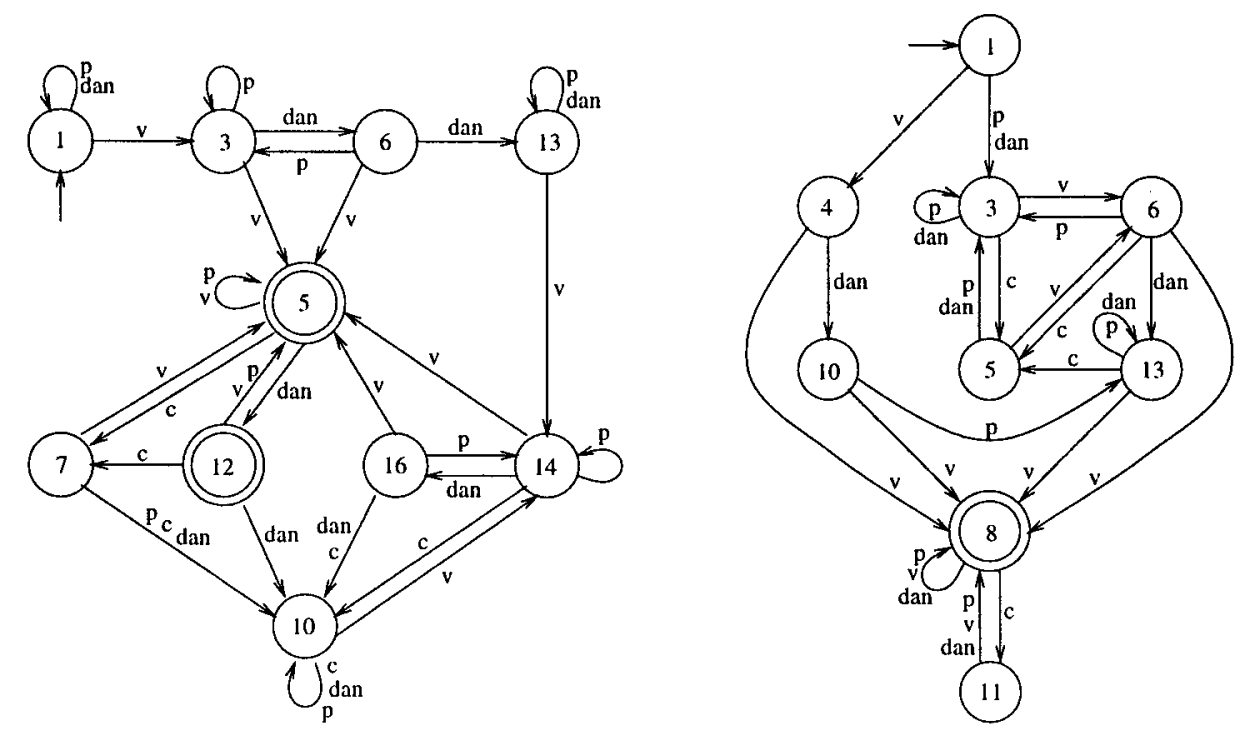

Figure 3: Finite-state approximations for the grammar in figure 1 calculated with the finite-state calculus (left) and by Pereira and Wright's algorithm (right). 
$a b$ is disallowed. Thus an approximation based on Rimon and Herz's 'next' would be $a a^{*}+b b^{*}$, and an approximation based on conditions $1-6$ would be $(a+b)(a+b)$. (However, the approximation becomes exact when conditions $7-8$ are added.)

Both Pereira and Wright $(1991 ; 1996)$ and Rood (1996) start with the LR(0) characteristic machine, which they first 'unfold' (with respect to 'stacks' or 'paths', respectively) and then 'flatten'. The characteristic machine is defined in terms of dotted rules with transitions between them that are analagous to the conditions implied by formula 3 of section 3 . When the machine is flattened, $\epsilon$-transitions are added in a way that is in effect simulated by conditions 2 and 4. (Condition 1 turns out to be implied by conditions 2-4.) It can be shown that the approximation $L_{0}$ obtained by flattening the characteristic machine (without unfolding it) is as good as the approximation $L_{1-6}$ obtained by applying conditions 1-6 $\left(L_{0} \subseteq L_{1-6}\right)$. Moreover, if no nonterminal for which there is an $\epsilon$-production is used more than once in the grammar, then $L_{0}=L_{1-6}$. (The grammar in figure 1 is an example for which $L_{0} \neq L_{1-6}$; the approximation found in section 6 includes strings such as vvccrv which are not accepted by $L_{0}$ for this grammar.) It can also be shown that $L_{1-6}$ is the same as the result of flattening the characteristic machine for the same grammar modifed so as to fulfil the afore-mentioned condition by replacing the right-hand side of every $\epsilon$-production with a new nonterminal for which there is a single $\epsilon$-production.

However, there does not seem to be a simple correspondence between conditions 7-8 and the 'unfolding' used by Pereira and Wright or Rood: even some simple grammars such as ' $\mathrm{S} \rightarrow$ a $\mathrm{S}$ a $|\mathrm{b} S \mathrm{~b}| \epsilon$ ' are approximated differently by $1-8$ than by Pereira and Wright's and Rood's methods.

\section{Discussion and conclusions}

In the case of some simple examples (such as the grammar ' $\mathrm{S} \rightarrow \mathrm{a} \mathrm{S}$ b $\mid \epsilon$ ' used earlier) the approximation algorithm presented in this paper gives the same result as Pereira and Wright's algorithm. However, in many other cases (such as the grammar ' $\mathrm{S}$ $\rightarrow \mathrm{a} S \mathrm{a}|\mathrm{b} \mathrm{S} \mathrm{b}| \epsilon$ ' or the 18-rule grammar in the previous section) the results are essentially different and neither of the approximations is better than the other.

The new algorithm does not share the problem of Pereira and Wright's algorithm that certain rightlinear grammars give an intermediate automaton of exponential size, and it was possible to calculate a useful approximation fairly rapidly in the case of the 18-rule grammar in the previous section. However, it is not yet possible to draw general conclusions about the relative efficiency of the two procedures. Nevertheless, the new algorithm seems to have the advantage of being open-ended and adaptable: in the previous section it was possible to complete a difficult calculation by relaxing the conditions of formulae $7-$ 8 , and it is easy to see how those conditions might also be strengthened. For example, a more complicated version of formulae 7-8 might check two levels of recursive application of the same rule rather than just one level and it might be useful to generalise this to $n$ levels of recursion in a manner analagous to Rood's (1996) generalisation of Pereira and Wright's algorithm.

The algorithm also demonstrates how the general machinery of a finite-state calculus can be usefully applied as a framework for expressing and solving problems in natural language processing.

\section{References}

Grimley Evans, Edmund, George Kiraz, and Stephen Pulman. 1996. Compiling a PartitionBased Two-Level Formalism. COLING-96, 454459.

Herz, Jacky, and Mori Rimon. 1991. Local Syntactic Constraints. Second International Workshop on Parsing Technology (IWPT-2).

Kaplan, Ronald, and Martin Kay. 1994. Regular models of phonological rule systems. Computational Linguistics, 20(3): 331-78.

Kempe, Andé, and Lauri Karttunen. 1996. Parallel Replacement in Finite State Calculus. COLING96,622 .

Percira, Fernando, and Rebecca Wright. 1991. Finite-state approximation of phrase structure grammars. Proceedings of the 29th Annual Meeting of the Association for Computational Linguistics, 246-255.

Pereira, Fernando, and Rebecca Wright. 1996. Finite-State Approximation of Phrase-Structure Grammars. cmp-lg/9603002.

Raymond, Darrell, and Derick Wood. March 1996. The Grail Papers. University of Western Ontario, Department of Computer Science, Technical Report TR-491.

Rimon, Mori, and Jacky Herz. 1991. The recognition capacity of local syntactic constraints. ACL Proceedings, 5th European Meeting.

Rood, Cathy. 1996. Efficient Finite-State Approximation of Context Free Grammars. Proceedings of ECAI 96. 
Shieber, Stuart. 1985. Using restriction to extend parsing algorithms for complex-feature-based formalisms. Proceedings of the 23nd Annual Meeting of the Association for Computational Linguistics, 145-152.

Van Noord, Gertjan. 1996. FSA Utilities: Manipulation of Finite-State Automata implemented in Prolog. First International Workshop on Implementing Automata, University of Western Ontario, London Ontario, 29-31 August 1996.

Watson, Bruce. 1996. Implementing and using finite automata $t$ olkits. Procuedings of ECAI 96. 